\title{
A comparison of the efficacy of microscopy versus rapid diagnostic testing in sub-Saharan Africa
}

\author{
Lauren Prufer (Meds 2016) \\ Faculty Reviewer: Dr S M Mansour Haeryfar, PhD (Department of Microbiology \& Immunology)
}

\begin{abstract}
Despite advances in treatment and diagnosis, malaria continues to cause a significant number of deaths, particularly in African children. ${ }^{1}$ Optimized diagnostic and follow-up testing is required for appropriate treatment and eventual eradication of malarial disease in sub-Saharan Africa. Microscopy is compared to rapid diagnostic testing (RDT) for use in a low-resource setting.
\end{abstract}

\section{INTRODUCTION}

Malaria is a curable disease caused by the infection of red blood cells with the parasite Plasmodium, a protozoan transmitted between carriers via bites from infected Anopheles mosquitos. According to the World Health Organization (WHO), malaria is responsible for between 473000 and 789000 deaths worldwide per year. ${ }^{1}$ Most deaths occur in children living in Africa, though elderly individuals, immunocompromised pregnant women and previously unexposed travellers are also at increased risk of experiencing severe disease in endemic areas. ${ }^{1,2}$ Chronic infection with the parasite results in partial immunity; individuals infected for the first time are the most vulnerable to complications of the disease, whereas those with chronic infections often become asymptomatic carriers. ${ }^{1}$ Malarial illness typically presents with fever, chills and vomiting, making its differential diagnosis difficult , and may result in severe complications or death if left untreated. ${ }^{2}$ Common complications observed in children include anemia, metabolic acidosis and cerebral malaria, whereas adults with severe disease are more likely to develop multi-organ failure. ${ }^{1}$ Childhood infection with malarial parasites has also been associated with increased prevalence of chronic anemia and status epilepticus in Africa. ${ }^{3,4}$ Thus, access to accurate and reliable diagnostic technology could help to reduce the burden of malaria-associated deaths and complications. ${ }^{1-4}$ Further, lack of accessibility and incorrect use of diagnostic technology has resulted in overprescription of antimalarial drugs. ${ }^{2}$ Widespread use of artemisinin monotherapy, rather then the gold standard artemisinin combination therapy (ACT), and discontinuation of antimalarial drugs based on symptom resolution rather then clearance of infection have contributed to the development of artemisinin-resistant strains in some geographical areas. ${ }^{5}$ The widespread dissemination of resistant strains could be devastating as methods of identifying and treating drug-resistant malarial strains have not yet been developed. ${ }^{5}$ Improved accessibility to accurate malaria diagnosis in sub-Saharan Africa is necessary for elimination of malaria as it ensures that individuals presenting with fever are treated appropriately, thus reducing overprescription and transmission to more vulnerable populations.
Though several tests exist for the diagnosis of malaria, there are many challenges to implementing them in sub-Saharan Africa. Previous efforts to eliminate malaria in this setting have failed despite the availability of diagnostic testing. Possible barriers to implementation may include poor infrastructure maintenance, lack of trained microscopy personnel, expiration or degradation of testing materials caused by heat and humidity, high financial costs and lack of quality control measures. In order to increase accessibility to testing in this setting, tests need to be sensitive and specific, but also easy to use, able to provide results rapidly and tolerant of poor storage conditions. This article will discuss the benefits and drawbacks of two commonly used technologies: microscopy and rapid diagnostic testing (RDT).

\section{MICROSCOPY}

Microscopy with Giemsa staining is considered the gold standard for diagnosis of malaria. ${ }^{6}$ Several different methods of microscopy are currently available. Thick blood smear microscopy examines a larger volume of blood and is thus useful for determining whether the parasite is present. ${ }^{6}$ Thin blood smear microscopy is less sensitive because a smaller volume of blood is used, but is more effective at determining parasite species: as such, it gives valuable information about a patient's risk for complications and the appropriate course of action. ${ }^{6}$

Microscopy performed in a clinical setting with skilled personnel is the most sensitive form of testing available for malaria diagnosis; detection of parasitemia can occur at concentrations of 4 to 20 parasites per $\mu \mathrm{L} .{ }^{6}$ However, this level of sensitivity is achievable only by experienced microscopists with adequate supplies and training. ${ }^{7,8}$ Field studies in Africa have demonstrated that parasitemia levels as high as 50 to 100 parasites per $\mu \mathrm{L}$ may be required for detection of malaria in local laboratory settings. ${ }^{7,8}$ Several studies conducted in febrile patients diagnosed with malaria have found poor agreement between regular microscopy and research microscopy and suggest that antimalarial overprescription rates may approach $50 \%$ in some areas. ${ }^{9}$ Sensitivity and specificity of regular microscopy was also found to vary considerably among different facilities, suggesting that the efficacy of microscopy is dependent upon the expertise of the microscopist. ${ }^{9,10}$ Several studies have been conducted in an African setting comparing routine microscopy to research microscopy. Results suggest that routine microscopy performs poorly in low-resource settings, with one trial finding that positive tests were confirmed by research microscopy only $43 \%$ of the time and the other, a study conducted in Dar es Salaam, finding only a $47 \%$ specificity for routine microscopy. ${ }^{2,10}$ It is therefore not surprising that a survey conduct- 
ed of clinicians in Tanzania found that only $41 \%$ considered malaria results from their local laboratory to be reliable. ${ }^{11}$ Reasons cited for poor laboratory performance include lack of equipment or stain, lack of training and low job motivation. ${ }^{11}$ Further, in both Ghana and Malawi, it was found that treatment is frequently administered despite negative test results. ${ }^{12}$ Thus, though microscopy can be highly effective at detecting parasitemia and differentiating between species of malaria, in the absence of appropriate resources and skilled technicians, it loses its diagnostic efficacy and may contribute to the overprescription of antimalarial drugs.

\section{RAPID DIAGNOSTIC TESTING}

Rapid diagnostic testing (RDT) is the use of immunochromographic assay with monoclonal antibodies to determine whether a blood sample contains protein markers specific for malaria. The test is simple to interpret and a result can be obtained in 5 to 20 minutes. Thus, use of these tests requires little training. Also, RDT kits are more transportable than microscopes and require no electricity, thus making them easier to implement in low-resource or rural settings. ${ }^{10}$ The same study that found low specificity for routine microscopy in clinics in Dar es Salaam reported the sensitivity and specificity of RDT to be $97 \%$ and $96.8 \%$, respectively. ${ }^{10}$ Implementing RDT in this setting significantly reduced false positive test rates from $43 \%$ in hospitals and $62 \%$ in health centers to $6 \%$ and $7 \%$, respectively, after RDT implementation. ${ }^{10}$ This observed true positive rate was similar to the rates of parasitemia in febrile patients found in a cross-sectional study of the region conducted at the same time. ${ }^{10}$ This suggests that the use of RDT could help to reduce overdiagnosis of malaria as well as the costs associated with overprescription of antimalarial drugs. A study conducted in Tanzania looked at the safety, measured by complication rate, of withholding antimalarial treatment based on RDT test results in 1000 febrile children with a mean age of 24 months. ${ }^{13}$ At 7 day follow-up, none of the 603 children who tested negative for malaria had malaria-related complications, though 4 were admitted to hospitals for other reasons, and 3 of the children who initially tested negative tested positive 2 , 4 or 7 days after initial testing. ${ }^{13}$ Children are at highest risk for malaria-related death in this region, and these results suggest that RDT could be a safe and cost-effective way to diagnose malaria and reduce overuse of antimalarial drugs in this population. ${ }^{5}$

It needs to be noted that there are several drawbacks to the use of RDTs. Firstly there are many products available on the market, which may use different antigens and have different sensitivities to parasitemia. Histidine-rich protein 2 (HRP2) has been demonstrated to have $P$ falciparum sensitivity of greater than $95 \%$ in several clinical trials, but results may vary considerably between kits and much lower sensitivities have been reported for other species. ${ }^{14-19}$ Assays using $P$ falciparum lactate dehydrogenase $(\mathrm{pLDH})$ or an aldolase, which are considered pan-malarial antigens, are also common, but some studies have reported sensitivities as low as $61 \%$ to $76 \%$ for non-falciparum species detection as well as decreasing test performance at lower levels of parasitemia. ${ }^{17-19}$ This means that asymptomatic carriers could go undiagnosed and untreated, resulting in continued propagation of the disease. As well, HRP2-specific assays are prone to false positives, as HRP-2 may take a long time to clear from the body or may remain elevated due to the presence of gametocytes, making this assay less useful for monitoring parasite clearance. ${ }^{20}$ This means clinicians may have to use one kit for diagnosis and a second for follow-up. Some of the observed poor results in trials may have been caused by product instability at temperatures greater then $30^{\circ} \mathrm{C}$ or by quality control issues during manufacturing. ${ }^{14}$ In order to address quality control issues, the WHO has created an initiative for quality assurance of RDTs that outlines an appropriate product testing protocol, monitors product quality and provides information to health care providers about the quality and efficacy of each product. ${ }^{21}$ This initiative has provided standards for specificity and sensitivity of RDTs as well as introduced quality control protocols to product manufacturing and testing, thus making RDT more robust and reliable in low-resource settings. ${ }^{21}$

\section{CONCLUSIONS}

Though expert microscopy remains the most sensitive and specific test for malaria diagnosis, the limited availability of appropriate lab infrastructure and expert microscopists in sub-Saharan Africa reduces its usefulness in this setting. Inaccurate malarial diagnosis may cause physicians to miss other potentially deadly causes of fever and may contribute to the overprescription of antimalarial agents and the evolution of drug-resistant malaria strains. RDTs have been shown to be both safe and cost-effective in the diagnosis of malaria, are simple to use and interpret and require little training or maintenance of infrastructure. Thus, RDTs may be a preferable method of malaria diagnosis in low-resource and rural settings.

\section{REFERENCES}

1. World Health Organization. Malaria fact sheet $\mathrm{N}^{\circ} 94$. Geneva $(\mathrm{CH})$ : World Health Organization; 2012

2. Strom GE, Tellevik MG, Fataki M, Langeland N, Blomberg B. No asymptomatic malaria parasitaemia found among 108 young children at one health facility in Dar es Salaam, Tanzania. Malar J. 2013;12:417.

3. Newton CR, Kariuki SM. Status epilepticus in sub-Saharan Africa: new findings. Epilepsia. 2013;54(6):50-3.

4. Magalhaes RJ, Clements AC. Mapping the risk of anaemia in preschool-age children: the contribution of malnutrition, malaria, and helminth infections in West Africa. PLoS Med. 2011;8(6):e1000438.

5. O'Brien C, Henrich PP, Passi N, Fidock DA. Recent clinical and molecular insights into emerging artemisinin resistance in Plasmodium falciparum. Curr Opin Infect Dis. 2011;24(6):570-7.

6. Ash LR, Orihel TC, Bosman A, Renganathan E, Rio F. Bench aids for the diagnosis of malaria infections. Geneva $(\mathrm{CH})$ : World Health Organization; 2000.

7. Milne LM, Kyi MS, Chiodini PL, Warhurst DC. Accuracy of routine laboratory diagnosis of malaria in the United Kingdom. J Clin Pathol. 1994;47(8):740-2.

8. Malaria diagnosis: memorandum from a WHO meeting. Bull World Health Organ. 1998;66(5):575-94.

9. Sleshi M, Animut A, Mohammed H, Medhin G, Kebede A. Malaria microscopy performance in self-presenting febrile patients at four health facilities in Fentale district of East Shewa, Ethiopia. Ethiop Med J. 2012;50(4):315-24.

10. Kahama-Maro J, D'Acremont V, Mtasiwa D, Genton B, Lengeler C. Low quality of routine microscopy for malaria at different levels of the health system in Dar es Salaam. Malar J. 2011;10(10):332.

11. Derua YA, Ishengoma DR, Rwegoshora RT, Tenu F, Massaga JJ, Mboera 


\section{DIAGNOSTIC REVIEW}

LE, Magesa SM. Users' and health service providers' perception on quality of laboratory malaria diagnosis in Tanzania. Malar J. 2011;10:78.

12. Pell C, Menaca A, Afrah NA, Manda-Taylor L. Prevention and management of malaria during pregnancy: findings from a comparative qualitative study in Ghana, Kenya and Malawi. Malar J. 2013;12(1):427.

13. d'Acremont V, Malila A, Swai N, Tillya R, Kahama-Maro J, Lengeler C, Genton B. Withholding antimalarials in febrile children who have a negative result for a rapid diagnostic test. Clin Infect Dis. 2010;51(5):506-11.

14. Wongsrichanalai C, Barcus MJ, Muth S, Sutamihardja A, Wernsdorfer WH. A review of malaria diagnostic tools: microscopy and rapid diagnostic test (RDT). Am J Trop Med Hyg. 2007;77(6 Suppl):119-27.

15. Fernando SD, Karunaweera ND, Fernando WP. Evaluation of a rapid whole blood immunochromatographic assay for the diagnosis of Plasmodium falciparum and Plasmodium vivax malaria. Ceylon Med J. 2004;49:7-11.

16. Murray CK, Bell D, Gasser RA, Wongsrichanalai C. Rapid diagnostic testing for malaria. Trop Med Int Health. 2003;8:876-83.

17. Pattanasin S, Proux S, Chompasuk D, Luwiradaj K, Jacquier P, Looareesuwan S, Nosten F. Evaluation of a new Plasmodium lactate dehydrogenase assay (OptiMAL-IT) for the detection of malaria. Trans R Soc Trop Med Hyg. 2003;97(6):672-4.

18. Iqbal J, Muneer A, Khalid N, Ahmed MA. Performance of the OptiMAL test for malaria diagnosis among suspected malaria patients at the rural health centers. Am J Trop Med Hyg. 2003:68(5): 624-8.

19. Farcas GA, Zhong KJ, Lovegrove FE, Graham CM, Kain KC. Evaluation of the Binax NOW ICT test versus polymerase chain reaction and microscopy for the detection of malaria in returned travelers. Am J Trop Med Hyg. 2003;69(6):589-92.

20. Ochola LB, Vounatsou P, Smith T, Mabaso ML, Newton CR. The reliability of diagnostic techniques in the diagnosis and management of malaria in the absence of a gold standard. Lancet Infect Dis. 2006;6(9):582-8.

21. World Health Organization. Good practices for selecting and procuring rapid diagnostic tests for malaria. Geneva $(\mathrm{CH})$ : World Health Organization; 2011. 